\title{
Charge instabilities near a Van Hove singularity
}

\author{
J. González \\ Instituto de Estructura de la Materia, Consejo Superior de Investigaciones Científicas, Serrano 123, 28006 Madrid, Spain
}

(Received 11 August 2000; published 9 January 2001)

\begin{abstract}
The charge instabilities of electron systems in the square lattice are analyzed near the Van Hove singularity by means of a Wilsonian renormalization group approach. We show that the method preserves the spin rotational invariance at all scales, allowing a rigorous determination of spin and charge instabilities of the $t$ $-t^{\prime}$ Hubbard model. Regarding the latter, repulsive interactions fall into two different universality classes. One of them has nonsingular response functions in the charge sector, while the other is characterized by the splitting of the Van Hove singularity. At the level of marginal perturbations, the Hubbard model turns out to be at the boundary between the two universality classes, while extended models with nearest-neighbor repulsive interactions belong to the latter class. In the case of open systems allowed to exchange particles with a reservoir, we show the existence of a range of fillings forbidden above and below the Van Hove singularity. This has the property of attracting the Fermi level in the mentioned range, as the system reaches its lowest energy when the Fermi energy is at the singularity.
\end{abstract}

DOI: 10.1103/PhysRevB.63.045114

PACS number(s): 71.10.Fd, 71.27.+a

\section{INTRODUCTION}

The effect of a Van Hove singularity near the Fermi surface of the $\mathrm{CuO}$ layers has been invoked recurrently to understand the unconventional properties of the high- $T_{c}$ materials. ${ }^{1,2}$ There have been several weak-coupling analyses of two-dimensional (2D) models of the Van Hove singularity, which have shown in particular that the $t-t^{\prime}$ Hubbard model may have a phase of $d$-wave superconductivity. ${ }^{3-6}$ The main problem that faces this proposal is that, although the system is likely to develop strong antiferromagnetic or superconducting correlations, the effective interactions grow large at low energies, so that it is not possible to discern rigorously the ground state of the model. A related issue concerns the fact that the superconducting correlations are enhanced like $\log ^{2} \varepsilon$, when the electron degrees of freedom are integrated out down to energy $\varepsilon$ near the Fermi surface. Recently, some understanding of the system has been attained by the use of refined renormalization group (RG) methods. $^{7-10}$ The analysis of the low-energy dynamics becomes then quite subtle, as the Fermi energy has proven to be a dynamical quantity susceptible itself of renormalization. ${ }^{11,12}$

The main purpose of this paper is to study the dynamics of the Fermi surface near a Van Hove singularity. Actually, the possible relevance of the strong correlations in the system could be objected by the need of a very fine adjustment of the Fermi energy at the singularity. We will show, however, that when the system is allowed to exchange particles with a reservoir it finds energetically more favorable to have the levels filled up to the position of the singularity. This leads to a natural pinning mechanism of the Fermi level over a certain range of fillings. 13,4

There is another effect that may be important, at fixed number of particles. It has been shown by Halboth and Metzner that the $t-t^{\prime}$ Hubbard model at the Van Hove filling should have an instability in its Fermi line leading to a spontaneous breakdown of the point group symmetry. ${ }^{14} \mathrm{We}$ will reproduce this effect in the form of a splitting of the levels of the two inequivalent saddle points of the 2D band, as a result of the renormalized interactions between electrons in the two hot spots. In general, we will show that the RG flows in the charge sector allow to distinguish two different universality classes for 2D electron systems near a Van Hove singularity. In one of them, the response functions do not show any instability under charge perturbations, while in the other the stable charge distribution is attained after the splitting of the Van Hove singularity. We will see, for instance, that extended Hubbard models with nearest-neighbor repulsive interactions belong to the latter universality class and that, for appropriate values of the couplings, the splitting becomes a sensible effect before the onset of any other instability of the system.

Our starting point will be a 2D model of electrons in the square lattice with nearest-neighbor hopping $t$ and nextnearest-neighbor hopping $t^{\prime}$. RG methods are most convenient for the description of the low-energy behavior of the interactions near the Van Hove singularity. In the RG approach, high-energy and low-energy electron modes are separated by an energy cutoff $\Lambda$, that is sent progressively towards the Fermi line as high-energy modes are integrated out in the RG process. ${ }^{15,16}$ When the Fermi level is at the Van Hove singularity, as shown in Fig. 1, most part of the low-energy states close to the Fermi line is concentrated around the saddle points at $(\pi, 0)$ and $(0, \pi)$, as these features are at the origin of the divergent density of states. Therefore, in building up the low-energy effective theory we may focus on two patches around the points $A$ and $B$, where the dispersion relation can be approximated by

$$
\varepsilon_{A, B}(\mathbf{k}) \approx \mp\left(t \mp 2 t^{\prime}\right) k_{x}^{2} a^{2} \pm\left(t \pm 2 t^{\prime}\right) k_{y}^{2} a^{2}
$$

$a$ being the lattice constant. From the RG point of view, the rest of modes far from the saddle points are irrelevant in the continuum limit $a \rightarrow 0$.

In fact, the effective action for the low-energy modes restricted to the region $\left|\varepsilon_{\alpha}(\mathbf{k})\right| \leqslant \Lambda$ can be written in the form 


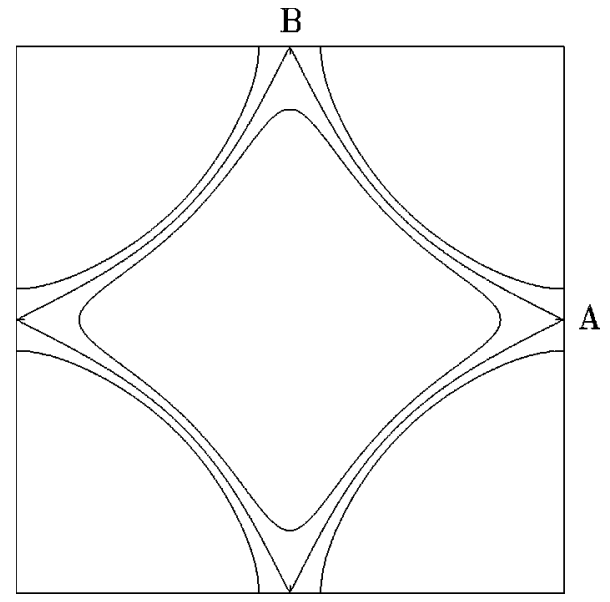

FIG. 1. Contour energy map for the $t-t^{\prime}$ Hubbard model about the Van Hove filling.

$$
\begin{aligned}
S= & \int d \omega d^{2} k \sum_{\alpha, \sigma}\left[\omega a_{\alpha, \sigma}^{+}(\mathbf{k}, \omega) a_{\alpha, \sigma}(\mathbf{k}, \omega)\right. \\
& \left.-\varepsilon_{\alpha}(\mathbf{k}) a_{\alpha, \sigma}^{+}(\mathbf{k}, \omega) a_{\alpha, \sigma}(\mathbf{k}, \omega)\right]-U \int d \omega d^{2} k \rho_{\uparrow}(\mathbf{k}, \omega) \\
& \times \rho_{\downarrow}(-\mathbf{k},-\omega),
\end{aligned}
$$

where $a_{\alpha, \sigma}\left(a_{\alpha, \sigma}^{+}\right)$are electron annihilation (creation) operators ( $\sigma$ labels the spin) and $\rho_{\uparrow}, \rho_{\downarrow}$ are the electron density operators. Under a change in the cutoff $\Lambda \rightarrow s \Lambda$, with a corresponding scaling of the frequency $\omega \rightarrow s \omega$ and the momenta $\mathbf{k} \rightarrow s^{1 / 2} \mathbf{k}$, one can check that the effective action remains scale invariant after an appropriate scale transformation of the electron modes, $a_{\alpha, \sigma} \rightarrow s^{-3 / 2} a_{\alpha, \sigma} \cdot{ }^{4}$

In writing the effective action (2) we have taken a local density-density interaction, like that of the Hubbard model. A most important point, however, is that in the process of renormalization other effective interactions may be generated as well, as long as they are compatible with the symmetries of the model. This issue will be reviewed in Sec. II, ending up with the proof that our Wilsonian RG scheme preserves the spin rotational invariance. Section III will be devoted to study the stability of the different distributions of the charge between the two hot spots, taking into account the behavior of the renormalized interactions. The stability of the location of the Fermi level around the Van Hove singularity will be discussed in Sec. IV, when the system is placed in contact with a charge reservoir. Finally, Sec. V will be devoted to conclusions and to comment on possible experimental realizations of our results.

\section{WILSONIAN RENORMALIZATION GROUP}

The Wilsonian RG approach, that has been recently applied to the investigation of many-body electron systems, ${ }^{15,16}$ provides a very efficient way of extracting the effective interactions of the low-energy theory. It represents an alternative to dealing with any kind of diagrammatic approximation built from the effective action (2), which has to suffer from severe infrared divergences. It is well known that the differ- ent susceptibilities of the model show logarithmic dependences on the cutoff $\Lambda$. In the case of the particle-hole susceptibility $\chi_{\mathrm{ph}}(\mathbf{p})$ and the particle-particle susceptibility $\chi_{\mathrm{pp}}(\mathbf{p})$ at small momentum $\mathbf{p}$, and the particle-hole susceptibility $\chi_{\mathrm{ph}}(\mathbf{q})$ at $\mathbf{q} \approx \mathbf{Q} \equiv(\pi, \pi)$, we have ${ }^{17}$

$$
\begin{gathered}
\chi_{\mathrm{ph}}(\mathbf{p}) \approx \frac{c}{2 \pi^{2} t} \log \left|\frac{\Lambda}{\varepsilon(\mathbf{p})}\right|, \\
\chi_{\mathrm{pp}}(\mathbf{p}) \approx \frac{c}{4 \pi^{2} t} \log ^{2}\left|\frac{\Lambda}{\varepsilon(\mathbf{p})}\right|, \\
\chi_{\mathrm{ph}}(\mathbf{p}+\mathbf{Q}) \approx \frac{c^{\prime}}{2 \pi^{2} t} \log \left|\frac{\Lambda}{t a^{2} \mathbf{p}^{2}}\right|,
\end{gathered}
$$

where $\quad c \equiv 1 / \sqrt{1-4\left(t^{\prime} / t\right)^{2}} \quad$ and $\quad c^{\prime} \equiv \log [(1$ $\left.\left.+\sqrt{1-4\left(t^{\prime} / t\right)^{2}}\right) /\left(2 t^{\prime} / t\right)\right]$.

When performing a RG calculation in the field theory approach, one computes the variation of the couplings under scale transformations by taking the derivatives of the above objects with respect to the cutoff. The feasibility of the RG method comes from the fact that, in general, the derivatives of the divergent diagrams do not depend themselves on the cutoff, what leads to the notion of scaling. In the present case, however, the derivative of the particle-particle susceptibility produces a contribution of the form $\log |\Lambda / \varepsilon(\mathbf{p})|$. This leads to an ill-defined computational procedure, as the argument of the logarithm requires an external ad hoc parameter for its definition. Otherwise stated, operators which receive contributions from particle-particle diagrams display, in general, cutoff dependences multiplied by a nonlocal, infrared divergent function of the external momenta. ${ }^{11}$ This is the fundamental problem when one tries to apply the RG program to the model of the Van Hove singularity, which, at present, seems to find a solution only by promoting the Fermi energy to a renormalized, scale-dependent variable. $^{11,12}$

Opposite to the field theory RG approach, the Wilsonian RG approach provides a better computational framework to deal with the above problem, as it makes a clear distinction of the operators which are renormalized in the particleparticle channel. The idea is to find the low-energy effective theory by identifying the operators that scale appropriately as the cutoff is sent to zero. This task is accomplished by performing a progressive integration of high-energy modes living in two thin shells of width $d \Lambda$, at distance $\Lambda$ in energy below and above the Fermi surface. In this process one keeps only operators which remain scale invariant, or which receive corrections at most of order $d \Lambda$, as the rest of the contributions vanish in the limit $\Lambda \rightarrow 0 .{ }^{15}$

Let us concentrate on the region around one of the saddle points, in which the two thin slices of width $d \Lambda$ look as shown in Fig. 2. The modes in the two slices build up the intermediate states in the corrections by particle-hole and particle-particle diagrams to the vertex functions of the theory. Focusing on the four-point function, we observe that such corrections are linear in $d \Lambda$ only in a reduced number 


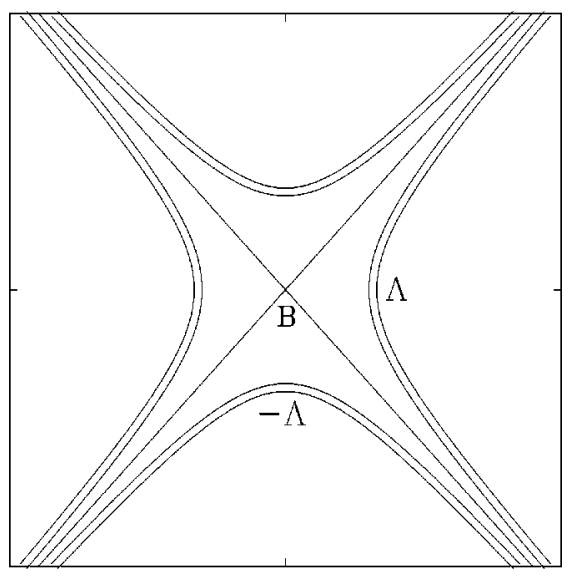

FIG. 2. Plot of the slices with high-energy states integrated in the renormalization group process.

of instances. Actually, contributions of order $d \Lambda / \Lambda$ arise for the same kinematics which do not make irrelevant the fourpoint function in Fermi liquid theory. ${ }^{15}$ They amount to three different possibilities, which are represented graphically in Fig. 3.

The BCS channel, that we denote by $V$, opens up when the momenta of the incoming particles add up to zero. At the one-loop level, for instance, it receives a contribution from the particle-particle diagram in Fig. 4. It is clear that, for each internal line with momentum $\mathbf{k}$ in the slice of width $d \Lambda$, the opposite momentum $-\mathbf{k}$ is also found among the high-energy states integrated over, so that the diagram is of order $\sim d \Lambda$. A similar argument shows that, when the sum of the momenta of the incoming particles is not zero, the set of available intermediate states is reduced to the intersection of two slices, displaced with respect to each other, and the phase space to build the diagram becomes of order $\sim(d \Lambda)^{2}$. Thus, in the Wilsonian RG approach the particle-particle diagram only renormalizes the vertex function for the precise kinematics of the BCS channel, while it produces irrelevant contributions for other choices of the external momenta. ${ }^{15}$

The forward-scattering channel $F$ is singled out in the vertex function when the momentum transfer along one of the fermion lines vanishes in the diagram. Technically, we may distinguish it from the exchange channel $E$, which arises when the momentum transfer between two lines connected by the interaction vanishes. It is clear anyhow that, when the incoming and outgoing particles have all the same spin, the respective channels $F_{\|}$and $E_{\|}$contribute with opposite sign to the same scattering amplitudes. It can be checked that all the corrections can be written in terms of the combination $F_{\|}-E_{\|}$, so that $E_{\|}$can be redefined away by introducing the coupling $\widetilde{F}_{\|}=F_{\|}-E_{\|}$.
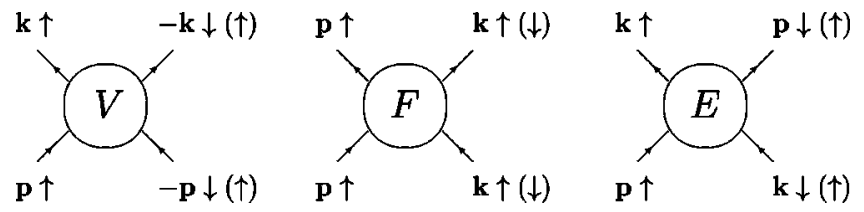

FIG. 3. Different channels that undergo renormalization in the Wilsonian approach.

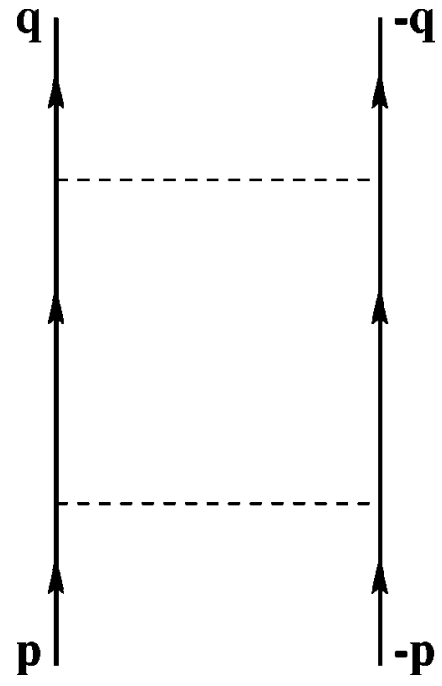

FIG. 4. Particle-particle diagram renormalizing the BCS channel at the one-loop level.

For incoming and outgoing particles with the same spin, all the diagrams shown in Fig. 5, with internal momenta in the slices of width $d \Lambda$ integrated over, produce a correction of order $\sim d \Lambda$ to the $\widetilde{F}_{\|}$coupling. Similarly, diagrams (a) and (b) in Fig. 5 are responsible for a renormalization of order $\sim d \Lambda$ of the $F_{\perp}$ coupling. This is a consequence of the fact that, for no matter how small momentum transfer, one can always build particle-hole excitations in the asymptotic region where two slices approach the Fermi line, as observed in Fig. 2.

On the other hand, when the vanishing momentum transfer takes place from one particle to another with different spin, we may still think of it as a different channel, that we call the $E_{\perp}$ exchange channel. ${ }^{18}$ In that case, a number of intermediate particle-hole excitations of order $\sim d \Lambda$ can be counted from the diagram in Fig. 6, which is the only one that renormalizes the $E_{\perp}$ channel.

It can be appreciated from the diagrams in Figs. 5 and 6 that the renormalization of the $F$ channel only depends on the $\widetilde{F}_{\|}$and $F_{\perp}$ couplings, as well as only $E_{\perp}$ couplings enter in the diagrams renormalizing the $E_{\perp}$ channel. On the other hand, the analysis of the instabilities of the model can be carried out in parallel, by using either set of couplings, together with the $V$ couplings. This is due to the fact that the $F$ couplings feed the correlations of the $z$ projection of the spin operators, while the $E_{\perp}$ couplings drive the correlations for

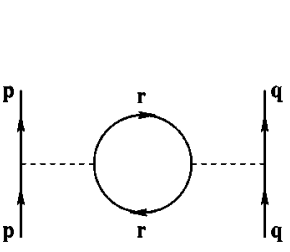

(a)

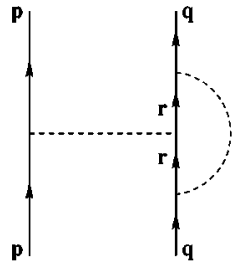

(b)

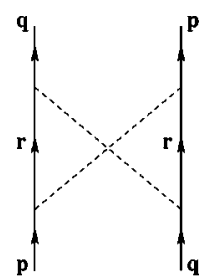

(c)
FIG. 5. Particle-hole diagrams renormalizing the $F$ channel at the one-loop level. 


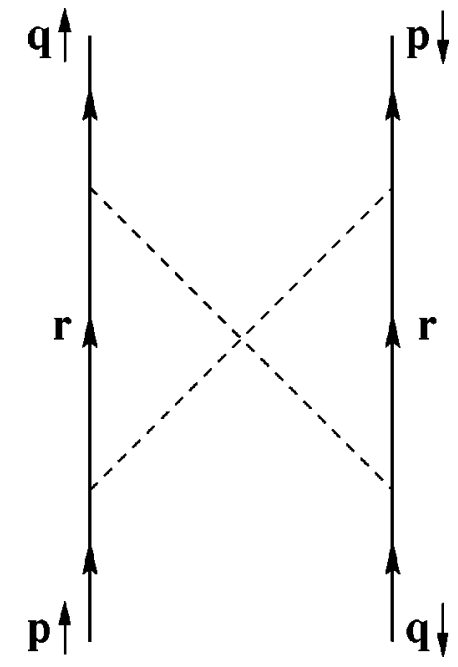

FIG. 6. Particle-hole diagram renormalizing the $E_{\perp}$ channel at the one-loop level.

the $x$ and $y$ components. The flow equations for the $F$ and the $E_{\perp}$ couplings have been studied in Refs. 12 and 7, respectively. It can be shown that the different phases that one obtains for the model (ferromagnetism, antiferromagnetism, superconductivity) do not depend on the use of one set of equations or the other. This relies on the key assumption of spin rotational invariance of our RG scheme, that we turn to check next.

It is possible to show that the response functions that measure the spin correlations for the $x, y$, and $z$ components of the spin are numerically equal, at each point of the RG flow, with a suitable choice of the bare couplings of the model. We deal in particular with the response functions at zero momentum, which measure the correlations of the operators

$$
S_{i}=\sum_{k} \sum_{\alpha=A, B} a_{\alpha, \sigma}^{+}(\mathbf{k}) \sigma_{\sigma \sigma^{\prime}}^{i} a_{\alpha, \sigma^{\prime}}(\mathbf{k}), \quad i=x, y, z .
$$

The following analysis can be also applied with complete similarity to the response functions at finite wave vector $\mathbf{Q}$ $\equiv(\pi, \pi)$.

Scaling equations for the response functions can be derived in the same fashion as for the renormalizable onedimensional models. ${ }^{19}$ The first-order contributions to the response function $R_{z}(\omega)$ for the $S_{z}$ operator are given in Fig. 7 . We introduce here a distinction between the interactions $F_{\text {intra }}$ and $E_{\text {intra }}$ for currents in the same saddle point and the interactions $F_{\text {inter }}$ and $E_{\text {inter }}$ between currents at different saddle points. After taking the derivative with respect to the cutoff and imposing the self-consistency of the diagrammatic expansion, we obtain

$$
\begin{aligned}
\frac{\partial R_{z}}{\partial \Lambda}= & -\frac{2 c}{\pi^{2} t} \frac{1}{\Lambda} \\
& +\frac{c}{\pi^{2} t}\left(\widetilde{F}_{\text {intra } \|}-F_{\text {intra } \perp}+\widetilde{F}_{\text {inter } \|}-F_{\text {inter } \perp}\right) \frac{1}{\Lambda} R_{z},
\end{aligned}
$$

where we have used the redefinition $\widetilde{F}_{\|} \equiv F_{\|}-E_{\|}$.

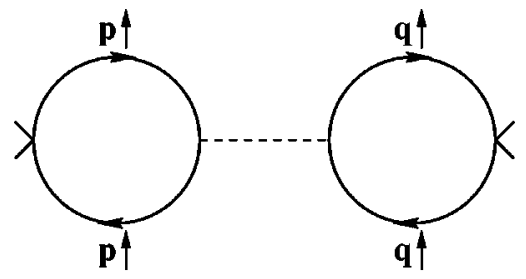

(a)

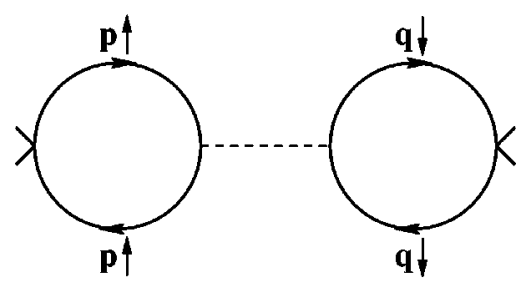

(b)

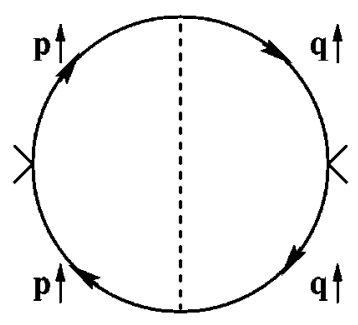

(c)

FIG. 7. First-order contributions to the correlator of the $S_{z}$ operator.

Similar scaling equations can be obtained for the response functions for the other projections of the spin, $R_{x}$ and $R_{y}$. In both cases, we have the first-order contribution shown in Fig. 8. The scaling equation for $R_{x}$, for instance, reads

$$
\frac{\partial R_{x}}{\partial \Lambda}=-\frac{2 c}{\pi^{2} t} \frac{1}{\Lambda}-\frac{c}{\pi^{2} t}\left(E_{\mathrm{intra} \perp}+E_{\mathrm{inter} \perp}\right) \frac{1}{\Lambda} R_{x} .
$$

From inspection of Eqs. (7) and (8), it turns out that $R_{x}$, $R_{y}$, and $R_{z}$ are identical as long as the constraints $F_{\text {intra }}$ $-\widetilde{F}_{\text {intra\| }}=E_{\text {intra } \perp}$ and $F_{\text {inter } \perp}-\widetilde{F}_{\text {inter } \|}=E_{\text {inter } \perp}$ are fulfilled at

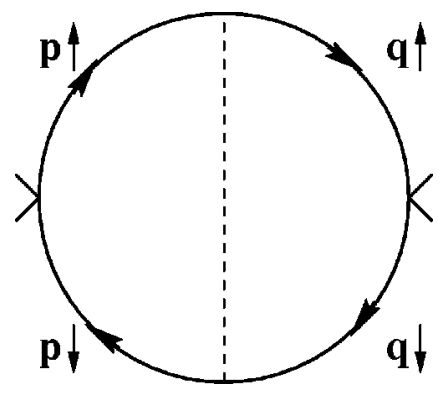

FIG. 8. First-order contribution to the correlators of the $S_{x}$ and $S_{y}$ operators. 
all the points of the RG flow. It can be easily seen that this is in fact the case provided that the initial values of the couplings satisfy both conditions.

The RG flow equations for the interactions in the forwardscattering channel can be obtained from Ref. 12. For the combinations $F_{\text {intra } \perp}-\widetilde{F}_{\text {intra } \|}$ and $F_{\text {inter } \perp}-\widetilde{F}_{\text {inter } \|}$, they read

$$
\begin{aligned}
& \Lambda \frac{\partial\left(F_{\text {intra } \perp}-\widetilde{F}_{\text {intra } \|}\right)}{\partial \Lambda} \\
& \quad=-\frac{1}{2 \pi^{2} t} c\left[\left(F_{\text {intra } \perp}-\widetilde{F}_{\text {intra } \|}\right)^{2}+\left(F_{\text {inter } \perp}-\widetilde{F}_{\text {inter } \|}\right)^{2}\right], \\
& \Lambda \frac{\partial\left(F_{\text {inter } \perp}-\widetilde{F}_{\text {inter } \|}\right)}{\partial \Lambda} \\
& \quad=-\frac{1}{\pi^{2} t} c\left(F_{\text {intra } \perp}-\widetilde{F}_{\text {intra } \|}\right)\left(F_{\text {inter } \perp}-\widetilde{F}_{\text {inter } \|}\right) .
\end{aligned}
$$

The RG equations in the exchange channel can be taken from Ref. 7. For $E_{\text {intra } \perp}$ and $E_{\text {inter } \perp}$ they have the form ${ }^{20}$

$$
\begin{gathered}
\Lambda \frac{\partial E_{\text {intra } \perp}}{\partial \Lambda}=-\frac{1}{2 \pi^{2} t} c\left(E_{\text {intra } \perp}^{2}+E_{\text {inter } \perp}^{2}\right), \\
\Lambda \frac{\partial E_{\text {inter } \perp}}{\partial \Lambda}=-\frac{1}{\pi^{2} t} c\left(E_{\text {intra } \perp} E_{\text {inter } \perp}\right) .
\end{gathered}
$$

It becomes manifest that, if $F_{\text {intra } \perp}-\widetilde{F}_{\text {intral }}=E_{\text {intra } \perp}$ and $F_{\text {inter } \perp}-\widetilde{F}_{\text {inter } \|}=E_{\text {inter } \perp}$ at the upper value of the cutoff, the two constraints are satisfied at any lower scale. Let us remark that this choice of initial conditions is actually quite reasonable, as it is what one would make by taking the bare values of the Hubbard interaction. We conclude that the spin rotational invariance of the model is preserved within our RG scheme, what is a rather remarkable result given the nontrivial flow of the RG equations. By taking the initial conditions $F_{\text {intra } \perp}-\widetilde{F}_{\text {intral }}>0$ and $F_{\text {inter } \perp}-\widetilde{F}_{\text {inter } \|}>0$, we observe that these combinations flow to strong coupling at low energies. In general, this kind of behavior leads to instabilities in the spin sector of the model, which have been studied by several authors. $3,7-9$

We have moreover the complementary flow equations

$$
\begin{aligned}
& \Lambda \frac{\partial\left(F_{\text {intra } \perp}+\widetilde{F}_{\text {intra } \|}\right)}{\partial \Lambda} \\
& \quad=\frac{1}{2 \pi^{2} t} c\left[\left(F_{\text {intra } \perp}+\widetilde{F}_{\text {intra } \|}\right)^{2}+\left(F_{\text {inter } \perp}+\widetilde{F}_{\text {inter } \|}\right)^{2}\right] \\
& \Lambda \frac{\partial\left(F_{\text {inter } \perp}+\widetilde{F}_{\text {inter } \|}\right)}{\partial \Lambda}=\frac{1}{\pi^{2} t} c\left(F_{\text {intra } \perp}+\widetilde{F}_{\text {intra\| } \|}\right)\left(F_{\text {inter } \perp}+\widetilde{F}_{\text {inter } \|}\right) .
\end{aligned}
$$

We assume that the bare couplings are such that $F_{\text {intra }}$ $+\widetilde{F}_{\text {intra } \|}>0$ and $F_{\text {inter } \perp}+\widetilde{F}_{\text {inter } \|}>0$. Under these conditions,

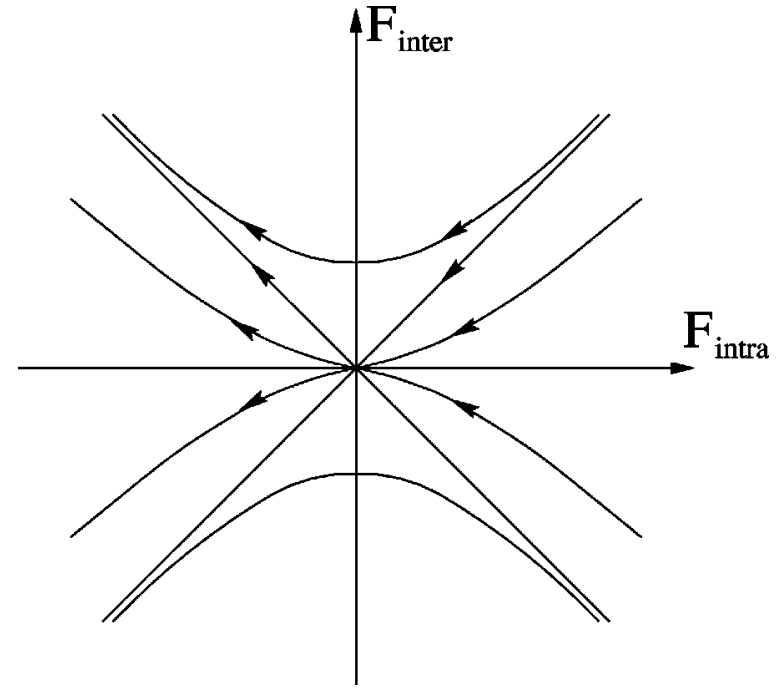

FIG. 9. Flow of the renormalized interactions in the $\left(\widetilde{F}_{\text {intral }}\right.$ $\left.+F_{\text {intra } \perp}, \widetilde{F}_{\text {inter } \|}+F_{\text {inter } \perp}\right)$ plane.

the flow may be attracted to two different regions, which characterize respective universality classes. When the initial couplings satisfy $F_{\text {intra } \perp}+\widetilde{F}_{\text {intra } \|}>F_{\text {inter } \perp}+\widetilde{F}_{\text {inter } \|}$, both combinations are renormalized to zero at low energies. The complete set of RG equations has then the asymptotic solution $\widetilde{F}_{\text {intra } \|} \approx-F_{\text {intra } \perp}$ and $\widetilde{F}_{\text {inter } \|} \approx-F_{\text {inter } \perp}$. Otherwise, when $F_{\text {intra } \perp}+\widetilde{F}_{\text {intra } \|}<F_{\text {inter } \perp}+\widetilde{F}_{\text {inter } \|}$ at the initial stage, the flow for these combinations of couplings becomes unstable, as shown in Fig. 9. The corresponding universality class is characterized by the asymptotic behavior $F_{\text {intraL }}+\widetilde{F}_{\text {intral }}$ $\approx-\left(F_{\text {inter } \perp}+\widetilde{F}_{\text {inter } \|}\right)$. This leads to important consequences in the charge sector, as we will see in what follows.

\section{CHARGE DYNAMICS BETWEEN HOT SPOTS}

The couplings $F_{\text {intra } \perp}+\widetilde{F}_{\text {intral }}$ and $F_{\text {inter } \perp}+\widetilde{F}_{\text {inter } \|}$ drive the interactions in the charge sector. They control the way in which the chemical potential is renormalized in the model. The chemical potential $\mu$ is introduced to fix the Fermi energy, but it gets corrections due to the charge present in the system. At the one-loop level, these corrections come from the diagrams in Fig. 10. The inspection of the kinematics of these diagrams shows that the charge in the system interacts through the combination of the couplings $F_{\text {intra }}+F_{\text {intral }}$ $-E_{\text {intra\| }}$ and $F_{\text {inter } \perp}+F_{\text {inter } \|}-E_{\text {inter } \|}$. These are actually what we have called $F_{\text {intra } \perp}+\widetilde{F}_{\text {intra\| }}$ and $F_{\text {inter } \perp}+\widetilde{F}_{\text {inter } \| \text {, respec- }}$ tively. The sum of all these couplings renormalizes to zero, in either of the two universality classes mentioned at the end of Sec. II. This means that, when the system is considered in isolation, its compressibility cannot be very different from that of the noninteracting model.

When the model falls in the universality class with the unstable flow $F_{\text {intra } \perp}+\widetilde{F}_{\text {intra } \|} \approx-\left(F_{\text {inter } \perp}+\widetilde{F}_{\text {inter } \|}\right)$, a mismatch in the filling levels of the two hot spots $A$ and $B$ may arise. This has been anticipated by Halboth and Metzner in a RG study of the $t-t^{\prime}$ Hubbard model, in the form of a 


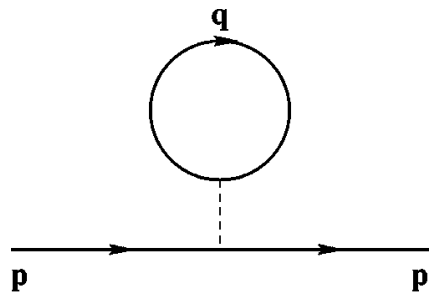

(a)

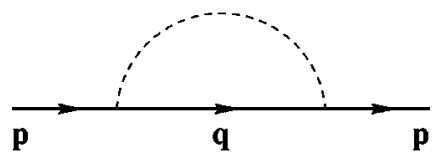

(b)

FIG. 10. Diagrams contributing to the electron self-energy at the one-loop level.

Pomeranchuk instability of the Fermi line. ${ }^{14}$ The same kind of effect can be obtained in our model as an instability in the response function $R_{A B}$ to perturbations in the difference of charge densities $n_{A}$ and $n_{B}$ at the two patches $A$ and $B$.

A scaling equation for the dynamic correlator $R_{A B}(\omega)$ of the operator $n_{A}-n_{B}$ can be derived with the same technique applied in Sec. II to the spin response functions. We obtain an expression of the form

$$
\begin{aligned}
\frac{\partial R_{A B}}{\partial \Lambda}= & -\frac{2 c}{\pi^{2} t} \frac{1}{\Lambda} \\
& +\frac{c}{\pi^{2} t}\left(F_{\text {intra } \perp}+\widetilde{F}_{\text {intra } \|}-F_{\text {inter } \perp}-\widetilde{F}_{\text {inter } \|}\right) \frac{1}{\Lambda} R_{A B} .
\end{aligned}
$$

From this equation, it can be checked that $R_{A B}$ develops a divergence at a finite value of the frequency whenever the bare couplings satisfy $F_{\text {intra } \perp}+\widetilde{F}_{\text {intra } \|}-F_{\text {inter } \perp}-\widetilde{F}_{\text {inter } \|}<0$. This is the signal that, when the Fermi level is nominally at the Van Hove singularity, an excess of charge develops in one of the hot spots over the other.

The precise nature of this instability can be clarified by performing a self-consistent solution of the SchwingerDyson equation

$$
G^{-1}=G_{0}^{-1}-\Sigma
$$

in our model with the two hot spots. The Fermi energy $\varepsilon_{F}$ in the full electron Green function $G$ is determined from the balance between the chemical potential $\mu$ in the free electron Green function $G_{0}$ and the corrections to it introduced by the electron self-energy. These corrections come at the one-loop level from the diagrams in Fig. 10, which depend in turn on the charge present in the system. Self-consistency is attained when the chemical potential after such renormalization matches the highest occupied level.

To study the interaction between the charge in the two hot spots, we model each of them by a singular density of states of the form

$$
n(\varepsilon)=-\frac{1}{\Lambda} \log (|\varepsilon| / \Lambda), \quad-\Lambda<\varepsilon<\Lambda .
$$

Furthermore, for the same nominal chemical potential $\mu$ of the system, we introduce two independent Fermi levels $\varepsilon_{A}$ and $\varepsilon_{B}$ for the respective hot spots. The Schwinger-Dyson equation referred to these two variables splits then in two equations of the form

$$
\begin{aligned}
\varepsilon_{A}= & \mu-\int_{-\Lambda}^{\varepsilon_{A}} d \varepsilon\left[F_{\text {intra } \perp}(\varepsilon)+\widetilde{F}_{\text {intra } \|}(\varepsilon)\right] n(\varepsilon) \\
& -\int_{-\Lambda}^{\varepsilon_{B}} d \varepsilon\left[F_{\text {inter } \perp}(\varepsilon)+\widetilde{F}_{\text {inter } \|}(\varepsilon)\right] n(\varepsilon), \\
\varepsilon_{B}= & \mu-\int_{-\Lambda}^{\varepsilon_{B}} d \varepsilon\left[F_{\text {intra } \perp}(\varepsilon)+\widetilde{F}_{\text {intra } \|}(\varepsilon)\right] n(\varepsilon) \\
& -\int_{-\Lambda}^{\varepsilon_{A}} d \varepsilon\left[F_{\text {inter } \perp}(\varepsilon)+\widetilde{F}_{\text {inter } \|}(\varepsilon)\right] n(\varepsilon),
\end{aligned}
$$

where we have introduced renormalized vertices in place of the four-point interactions in Fig. 10. We remark that $\varepsilon_{A}$ and $\varepsilon_{B}$ are measured in the reference frames in which the dependence of the density of states is fixed by Eq. (17). Thus, the fact that $\varepsilon_{A}$ and $\varepsilon_{B}$ may be nominally different after renormalization is just a consequence of that convention. The physical picture is however the opposite, namely that the one-particle levels are shifted to higher energy by a different amount in each of the two hot spots, up to a point in which the respective Fermi levels reach the common chemical potential.

It can be checked that, in the phase with the stable flow $F_{\text {intra } \perp}+\widetilde{F}_{\text {intra\| }}>F_{\text {inter } \perp}+\widetilde{F}_{\text {inter\| } \|}$ Eqs. (18) and (19) only admit a single solution with $\varepsilon_{A}=\varepsilon_{B}$. However, for couplings falling in the universality class with the unstable flow, together with that solution we find another which has different filling levels for the two hot spots. A plot of the filling levels versus the total charge in the system is represented in Fig. 11 , for the particular bare values $F_{\text {intra } \perp}=\Lambda, F_{\text {inter } \perp}=2 \Lambda$. We have found that the solution with $\varepsilon_{A} \neq \varepsilon_{B}$ turns out to have always the lowest energy. The physical interpretation of these results is that, due to the mismatch in the repulsive interaction, the one-particle levels are shifted upwards with higher strength in one of the hot spots than in the other, so that the common Fermi energy becomes placed below one of the saddle points and above the other. The lowest-energy solution describes therefore the splitting of the Van Hove singularity, in correspondence with the spontaneous breakdown of the tetragonal symmetry found by Halboth and Metzner.

If one were to take the nominal couplings of the Hubbard model as the bare interactions in the RG approach, this would lead to the initial condition $F_{\text {intra } \perp}=F_{\text {inter } \perp}=U$, with the rest of $F$ couplings equal to zero. Thus, the Hubbard model is placed right at the boundary between the region of unstable flow and the phase in which the $F_{\text {intra } \perp}+\widetilde{F}_{\text {intrall }}$ and $F_{\text {inter } \perp}+\widetilde{F}_{\text {inter } \|}$ couplings are renormalized to zero. The slightest perturbation by any irrelevant operator may drive 


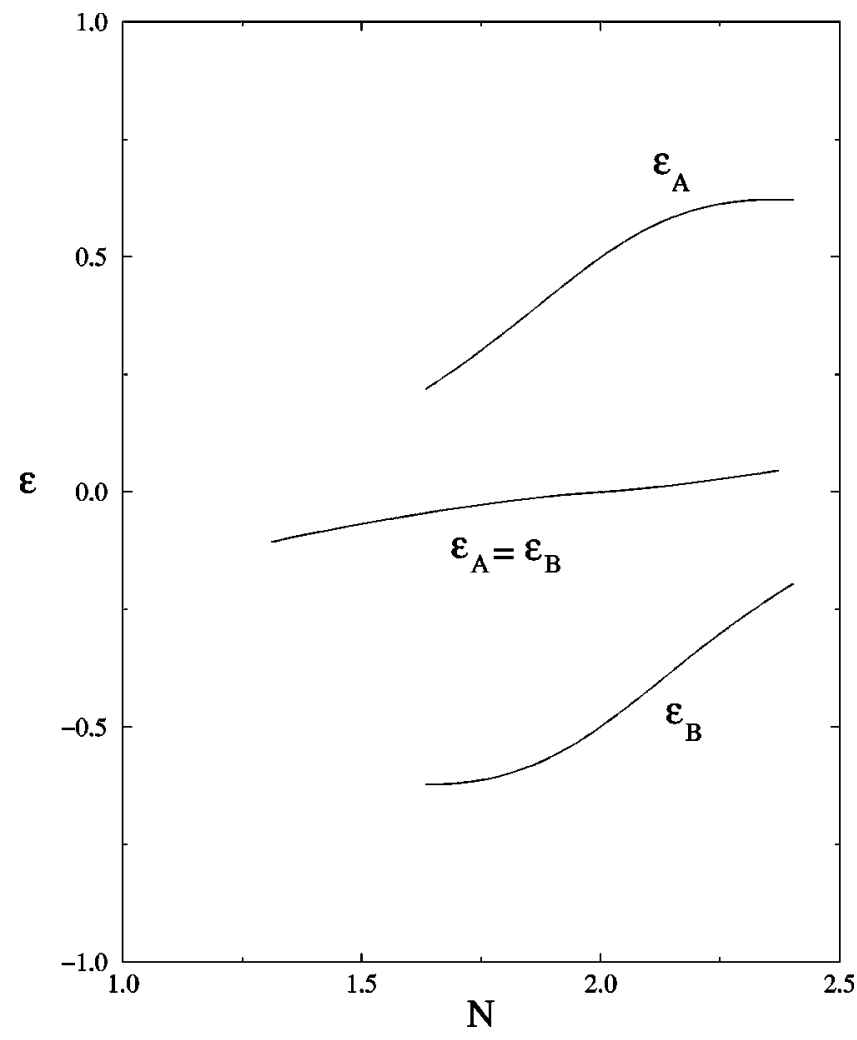

FIG. 11. Filling levels in the two hot spots versus total charge $N$. Middle curve: solution with even distribution of the charge. Upper and lower curves: uneven filling levels of the solution corresponding to the splitting of the Van Hove singularity.

the system to either of the two phases, and the work of Halboth and Metzner ${ }^{14}$ shows indeed that the Hubbard model in particular falls in the universality class with the charge instability.

However, the splitting of the Van Hove singularity is not a universal feature of $2 \mathrm{D}$ electron systems. There may exist models that lead instead to the initial condition $F_{\text {intra }}$ $+\widetilde{F}_{\text {intra } \|}>F_{\text {inter } \perp}+\widetilde{F}_{\text {inter } \|}$. According to Eqs. (13), (14), and (15), these models only have a weak, nonsingular response to any charge perturbation, and they do not show therefore the splitting of the Van Hove singularity. In general, whether one universality class or the other is realized depends on the particular details of the microscopic model. For an extended Hubbard model with nearest-neighbor interaction $V$, for instance, the assignment of bare couplings made from the nominal interactions in the model gives $F_{\text {intra } \perp} \sim U+4 V$, $F_{\text {inter } \perp} \sim U+4 V, \widetilde{F}_{\text {inter } \|} \sim 8 V$. In this case, the coupling driving the charge instability becomes $F_{\text {inter } \perp}+\widetilde{F}_{\text {inter } \|}-F_{\text {intra } \perp}$ $-\widetilde{F}_{\text {intral }} \sim 8 \mathrm{~V}$. It turns out that the model with extended attractive interaction $V<0$ fulfills the above condition for a nonsingular response. On the other hand, the model with extended repulsive interaction $V>0$ falls in the universality class with the charge instability. The tendency to the splitting of the Van Hove singularity is more pronounced in this case as the strength of the repulsive interaction $V$ is increased.

We have to bear in mind, though, that the energies at which the response to a charge perturbation becomes unstable may be below the scale at which other instabilities open up in the $t-t^{\prime}$ model. From Eq. (15), the charge instability arises at the point in which the renormalized coupling $F_{\text {inter } \perp}+\widetilde{F}_{\text {inter } \|}-F_{\text {intra } \perp}-\widetilde{F}_{\text {intra } \|}$ diverges. This happens at a frequency

$$
\begin{aligned}
\omega \approx & \Lambda \exp \left(-2 \pi^{2} t /\left\{c \left[F_{\text {inter } \perp}(\Lambda)+\widetilde{F}_{\text {inter } \|}(\Lambda)\right.\right.\right. \\
& \left.\left.\left.-F_{\text {intra } \perp}(\Lambda)-\widetilde{F}_{\text {intra } \|}(\Lambda)\right]\right\}\right)
\end{aligned}
$$

When $c>c^{\prime}$, the response functions at vanishing momentum dominate over those for perturbations with finite wave vector $\mathbf{Q} \equiv(\pi, \pi) .^{7}$ Taking the expressions of $c$ and $c^{\prime}$ given after Eqs. (3) $-(5)$, this corresponds to values of $t^{\prime}$ above $\approx 0.276 t .^{21}$ In this region of the phase diagram, a strong instability leading to ferromagnetism should also be present, as it has been shown in Ref. 22. The mismatch between the densities of spin up and spin down electrons is driven by the coupling $F_{\text {intra } \perp}-\widetilde{F}_{\text {intra } \|}+F_{\text {inter } \perp}-\widetilde{F}_{\text {inter } \|}$. From Eqs. (9) and (10) it is seen that the response leading to ferromagnetism becomes singular at the scale

$$
\omega_{\mathrm{FM}} \approx \Lambda \exp \left\{-\pi^{2} t /(c U)\right\} .
$$

Comparing with Eq. (20), we conclude that the splitting of the Van Hove singularity takes place prior to any other instability in the extended Hubbard model for $c>c^{\prime}$ and $4 \mathrm{~V}$ $>U$. For values $V<U / 4$ the comparison is more uncertain since, as we have pointed out before, the effect of irrelevant operators cannot be dismissed in that regime, specially in the limit $t^{\prime} \rightarrow 0.5 t, c \rightarrow \infty$. It is therefore likely a tight competition between the ferromagnetic and the charge instability for such small values of $V$.

For $c^{\prime}>c$, the tendency towards a spin-density-wave instability prevails over any other instability in the spin sector. In this regime, backscattering and Umklapp interactions become stronger as the nesting condition $t^{\prime}=0$ is approached. Besides, the scaling equation governing the spin-densitywave instability has the same structure that Eqs. (7) and (8), but with the coefficient $c^{\prime}$ instead of $c .^{7}$ The scale at which the response leading to the spin-density-wave becomes singular is

$$
\omega_{\mathrm{SDW}} \approx \Lambda \exp \left\{-\pi^{2} t /\left(c^{\prime} U\right)\right\} .
$$

Then, we observe that the formation of the spin-densitywave takes place before the onset of the charge instability for $4 c V<c^{\prime} U$, while the latter prevails in the opposite case.

Coming back to the particular case of the Hubbard model with purely on-site interaction, the charge instability is definitely excluded in favor of other instabilities for small values of $t^{\prime}$ below $\approx 0.276 t$. This is also the region of the phase diagram in which $d$-wave superconductivity is likely to occur, due to the Kohn-Luttinger mechanism. ${ }^{4,5}$ From inspection of Eqs. (20) and (22) it is clear that, as $t^{\prime} \rightarrow 0$ and $c^{\prime}$ $\rightarrow \infty$, the energy scale of the formation of the spin-densitywave becomes much higher than the scale at which the splitting of the Van Hove singularity takes place. Only for values of $t^{\prime}$ above $\approx 0.276 t$ there is a close competition between the spin and the charge instabilities, which requires to be resolved a more precise analysis than that carried out in terms of the marginal perturbations. 


\section{CHARGE DYNAMICS IN CONTACT WITH CHARGE RESERVOIR}

We have seen that a universal feature of our Wilsonian RG scheme is that the coupling to the total charge of the system is renormalized to zero when the Fermi level approaches the Van Hove singularity. This is just a consequence of the strong screening processes that arise due to the divergent density of states. That property does not have any sensible effect for a closed system with constant number of particles, since the Fermi energy can only be a monotonous function of the total charge. However, if the system is instead at fixed chemical potential, important effects may be derived from the mentioned result. The description at fixed chemical potential has to do with the situation in which the system is in contact with a charge reservoir, that has a much larger content of particles and is less susceptible to changes in its Fermi energy. This may be also the relevant situation for the physics of the high- $T_{c}$ materials, regarding the interaction of the $\mathrm{CuO}$ layers with the rest of the perovskite structure.

When the system does not have a fixed number of particles, the Fermi energy displays a nontrivial dynamics which tends to pin it to the Van Hove singularity. ${ }^{13,4}$ In the RG framework it has been shown that the Fermi energy, taken as a running parameter dependent on the high-energy cutoff, has a stable fixed-point very close in energy to the Van Hove singularity. ${ }^{4}$ From the physical point of view, this leads to the important consequence that a certain range of fillings should be forbidden above and below the singularity. The prediction is that, for nominal values of the chemical potential in that range, the Fermi energy is led to the fixed-point at the singularity. Only for lower or higher values of the chemical potential away from the region of attraction one may recover the regular evolution of the Fermi energy upon filling.

The pinning mechanism can be best understood by solving the Schwinger-Dyson equation for the model in contact with a system with large but constant density of states. The latter has then a Fermi energy much less sensitive to changes in the total number of particles, what amounts in practice to imposing the condition of fixed chemical potential in the part of the system with the Van Hove singularity. As in the preceding section, the Schwinger-Dyson equation written for the Fermi energy expresses how the filling level for each of the systems is renormalized by the shift of the one-particle levels to higher energies due to the repulsive interaction. The self-consistent dependence of this effect on the charge and the strength of the renormalized interactions leads to the unconventional dynamics of the Fermi level near the Van Hove singularity.

We model the system with the Van Hove singularity by taking the density of states

$$
n^{(1)}(\varepsilon)=-\frac{1}{\Lambda} \log (|\varepsilon| / \Lambda), \quad-\Lambda<\varepsilon<\Lambda
$$

For the system with large but constant density of states, we take a dependence of the form

$$
n^{(2)}(\varepsilon)=\frac{\alpha}{\Lambda}, \quad-\beta \Lambda<\varepsilon
$$

We assume that, in the first system, the coupling to the total charge, $\widetilde{F}_{\text {intral }}+F_{\text {intra } L}+\widetilde{F}_{\text {inter } \|}+F_{\text {inter } \perp}$, is renormalized near the singularity according to Eqs. (13) and (14). On the other hand, the interaction between particles in the charge reservoir is scale independent and we suppose that it can be parametrized by a constant coupling $F_{0}$ in the forwardscattering channel.

As in the preceding section, we introduce a common chemical potential $\mu$ for the two systems, which enforces the condition of thermodynamic equilibrium between them. The Schwinger-Dyson equation gives rise to the following pair of nonlinear equations for the respective filling levels $\varepsilon_{F 1}$ and $\varepsilon_{F 2}$ of the two systems

$$
\begin{gathered}
\varepsilon_{F 1}=\mu-\int_{-\Lambda}^{\varepsilon_{F 1}} d \varepsilon F(\varepsilon) n^{(1)}(\varepsilon)-g_{\mathrm{fwd}} \int_{-\beta \Lambda}^{\varepsilon_{F 2}} d \varepsilon n^{(2)}(\varepsilon), \\
\varepsilon_{F 2}=\mu-F_{0} \int_{-\beta \Lambda}^{\varepsilon_{F 2}} d \varepsilon n^{(2)}(\varepsilon)-g_{\mathrm{fwd}} \int_{-\Lambda}^{\varepsilon_{F 1}} d \varepsilon n^{(1)}(\varepsilon),
\end{gathered}
$$

where $F \equiv \widetilde{F}_{\text {intral }}+F_{\text {intra } \perp}+\widetilde{F}_{\text {inter } \|}+F_{\text {inter } \perp}$ and we have introduced a coupling constant $g_{\text {fwd }}$ that parametrizes the repulsion exerted on one of the systems by the charge present in the other.

We stress once more that, in the above equations, $\varepsilon_{F 1}$ and $\varepsilon_{F 2}$ are measured in the reference frames in which the dependences $n^{(1)}(\varepsilon)$ and $n^{(2)}(\varepsilon)$ are fixed by Eqs. (23) and (24). As remarked in the preceding section, the physical picture is however that the one-particle levels are renormalized to higher energy by a different amount in each of the systems, so that both Fermi levels match at the end the common chemical potential.

The coupled set of equations (25) and (26) gives rise to nontrivial physical effects, as a consequence of the nonlinearities introduced by the divergent density of states $n^{(1)}(\varepsilon)$ and the renormalization of $F(\varepsilon)$ close to the Van Hove singularity. It is interesting, for instance, to solve for the location of $\varepsilon_{F 1}$ and $\varepsilon_{F 2}$ in terms of the total charge $N$ in the two systems, given by

$$
N=\int_{-\Lambda}^{\varepsilon_{F 1}} d \varepsilon n^{(1)}(\varepsilon)+\int_{-\beta \Lambda}^{\varepsilon_{F 2}} d \varepsilon n^{(2)}(\varepsilon) .
$$

The most remarkable effect is that there is not a one-to-one correspondence between $N$ and the respective filling levels $\varepsilon_{F 1}$ and $\varepsilon_{F 2}$. The different branches of the solution are represented in Fig. 12 for the particular values $F(\Lambda)=F_{0}$ $=4 \Lambda, c / \pi^{2}=0.2$, and $g_{\text {fwd }}=3 \Lambda$. The parameter $\beta$ has been chosen equal to 3.0, and $\alpha$ has been set equal to 4.0, according to the idea of having a large density of states in the second of the systems.

At low values of $N$, the filling of the first system with the Van Hove singularity proceeds in a regular way, with a monotonous increase of $\varepsilon_{F 1}$. There is a point, however, above which two other locations of $\varepsilon_{F 1}$ become possible, closer to 


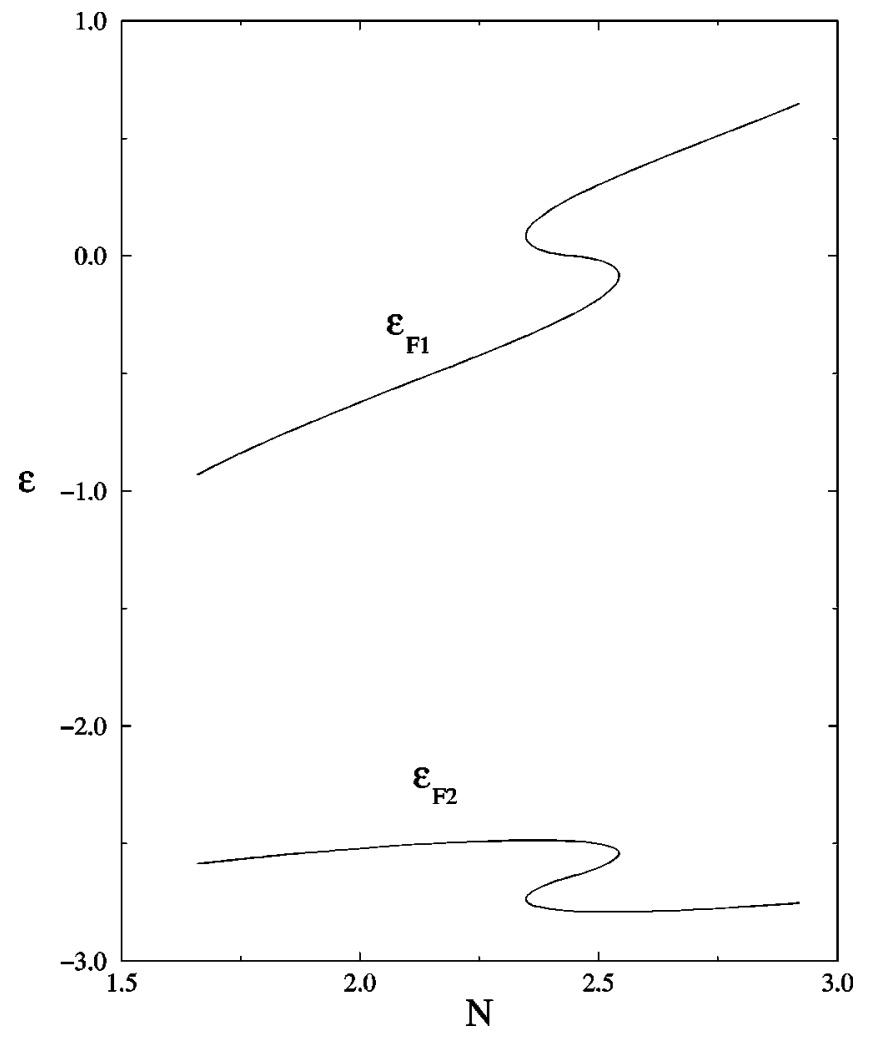

FIG. 12. Self-consistent solutions for the respective filling levels $\varepsilon_{F 1}$ and $\varepsilon_{F 2}$ in the system with the Van Hove singularity and in the charge reservoir.

the singularity in the density of states at $\varepsilon=0$. In these instances, the corresponding filling level $\varepsilon_{F 2}$ in the second system suffers a decrease with respect to the expected value. It is interesting to discern what of the possible solutions is most favorable energetically. We have plotted in Fig. 13 the values of the total energy $E$ versus the total charge $N$. We see that the filling level closer to the Van Hove singularity gives always the lowest-energy configuration of the system.

The result that turns out to be valid under very general conditions is the existence of a certain range of filling levels that are forbidden above and below the Van Hove singularity. This is in agreement with previous analyses of the pinning of the Fermi level of electrons near a Van Hove singularity. ${ }^{13,4}$ The present study of the Schwinger-Dyson equation helps to clarify the mechanism involved in that effect. It happens that, for certain values of the charge $N$, this finds more favorable to fill the Fermi sea up to the Van Hove singularity, at the expense of the charge in the other system. In general, there is a critical value $N_{c 1}\left(N_{c 2}\right)$ of the total charge in which the filling level $\varepsilon_{F 1}$ jumps discontinuously from the regular evolution upon adding (removing) particles to a position much closer to the Van Hove singularity. This is in correspondence with the onset of attraction to the stable fixed-point found in the RG framework.

A last remark regarding the plot in Fig. 13 is that the abrupt change in the lowest energy of the system at $N_{c 1}\left(N_{c 2}\right)$ leads to phase separation for values of $N$ below (above) that critical value. It is clear, for instance, that for a certain range above $N_{c 2}$ the whole system lowers its energy

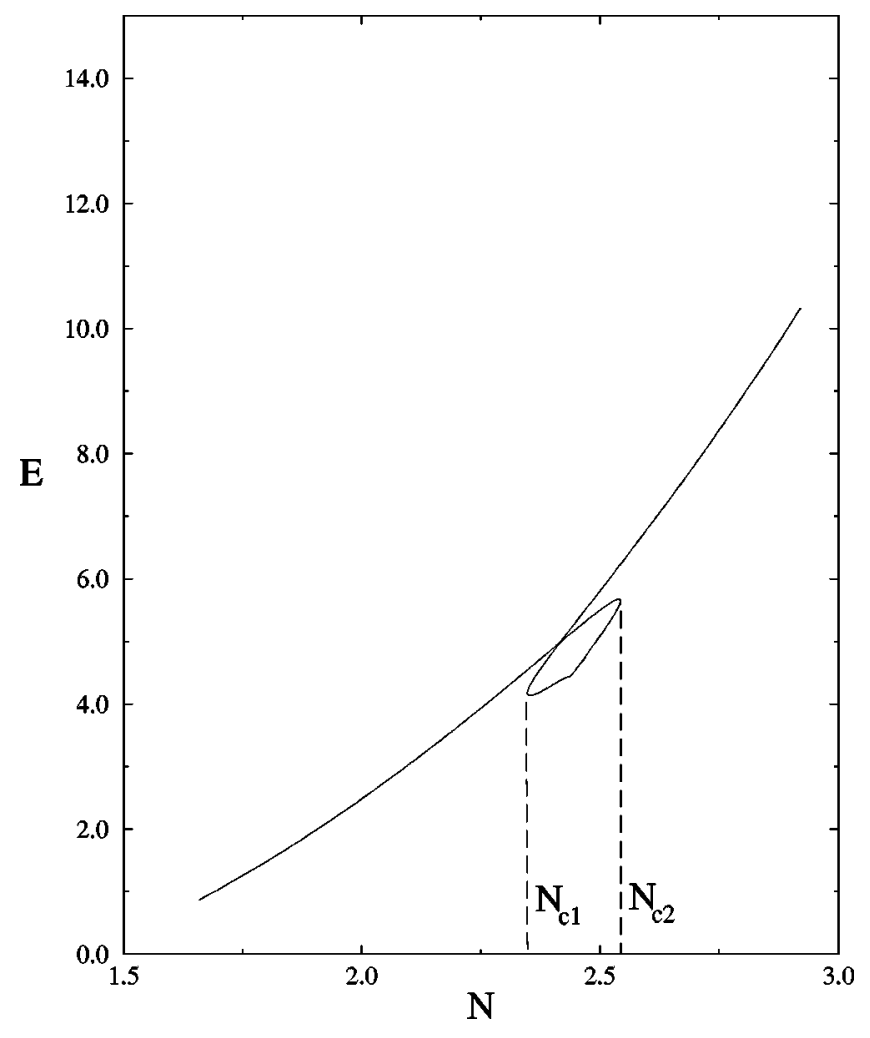

FIG. 13. Total energy of the solutions shown in Fig. 12 versus total charge $N$.

by splitting in two phases, one with a higher value of the charge density and the other with the density corresponding approximately to $N_{c 2} .{ }^{23}$ This reflects in another fashion that special stability is conferred to the system when the Fermi level is at the Van Hove singularity.

\section{CONCLUSIONS}

In this paper we have adopted a Wilsonian RG approach to discern the charge instabilities of 2D electron systems at the Van Hove filling. This kind of differential method of renormalization was implemented in Ref. 15 to discuss Fermi liquid theory in the context of the universality classes of interacting fermion systems. When applied to the system of electrons near the Van Hove singularity, we have seen that the method leads to a rigorous analysis of the instabilities in the spin and charge sectors.

It is well known that the main problem of dealing with the singular density of states in the RG framework is that it gives rise to harmful $\log ^{2} \Lambda$ divergences in the particle-particle diagrams. These divergences cannot be removed by RG methods in a standard fashion, as they actually point at the appearance of nonlocal operators that are infrared divergent in the limit of vanishing momentum at the singularity. In the differential RG approach, however, a careful analysis of the kinematics shows that, at least at the one-loop level, the divergences of the particle-particle diagrams only affect the BCS channel. ${ }^{15}$ The forward-scattering channel is only affected by conventional $\log \Lambda$ divergences. These are at the 
origin of potential instabilities in the correlators for the density of spin and charge, that can be now properly understood in the differential RG scheme.

An important property of our RG approach is that it allows to preserve the spin rotational invariance at all the steps of the RG process. In fact, of all the flows that we have described in the space of couplings, there is only a very reduced number of combinations that realize the mentioned invariance. This shows how stringent the Wilsonian approach can be by enforcing symmetry constraints to determine the low-energy effective theory.

Starting with bare repulsive interactions in the forward and exchange channels, we have seen that there are only two asymptotic low-energy behaviors consistent with the SU(2) spin invariance. One of them corresponds to the line $\widetilde{F}_{\|}$ $=F_{\perp}$, for all the forward-scattering couplings, and $E_{\perp}=0$, for all the exchange couplings. Under these conditions, all the $F$ couplings are renormalized to zero at low energies, and it is clear from Eqs. (7) and (8) that all the spin projections have equal dynamical correlations at all points of the flow. The other possibility corresponds to the choice $F_{\perp}-\widetilde{F}_{\|}$ $=E_{\perp}$ for all the couplings. In this case, these flow to a strong coupling regime with singular response functions in the spin sector. As discussed above, the $t-t^{\prime}$ Hubbard model has a low-energy behavior that falls within the latter class.

Turning to the charge instabilities, we have seen that the interaction between the electrons at the two inequivalent saddle points of the square lattice leads to two different universality classes for $2 \mathrm{D}$ electron systems near a Van Hove singularity. One of them corresponds to the RG flows below the bisector of the first quadrant in Fig. 9, for which $\widetilde{F}_{\text {intrall }}$ $+F_{\text {intra }}>\widetilde{F}_{\text {inter } \|}+F_{\text {inter } \perp \text {. In this class, both combinations }}$ of couplings are renormalized to zero at low energies, no response function in the charge sector displays singular behavior, and the instabilities may arise in the spin sector.

The other universality class corresponds to the unstable flows with $\widetilde{F}_{\text {intral }}+F_{\text {intra } L}<\widetilde{F}_{\text {inter } \|}+F_{\text {inter } \perp \text {, which lead to a }}$ singular response in the charge sector. We have shown that this phase is characterized on physical grounds by the splitting of the levels of the two inequivalent saddle points. This kind of instability has been found recently in a numerical RG study of the $t-t^{\prime}$ Hubbard model. ${ }^{14}$ A naive assignment of the bare couplings of the model gives $F_{\text {intra } \perp}=F_{\text {inter } \perp}=U$ and $\widetilde{F}_{\text {intra } \|}=\widetilde{F}_{\text {inter } \|}=0$, placing it right at the boundary between the two universality classes. However, the boundary is not itself stable and the effect of any irrelevant perturbation may break the balance in favor of either side. The findings of Ref. 14 show that this is indeed the case and that the Hubbard model has to belong to the universality class with the charge instability. In any event, our analysis makes clear that the singular response in the charge sector may develop before any instability in the spin sector only for values of $t^{\prime}$ above $\approx 0.276 t$. In that range, there is a competition with the ferromagnetic instability that is also known to open up in the model at the Van Hove filling. ${ }^{22}$

A feature common to both universality classes is that the coupling to the total charge, $\widetilde{F}_{\text {intral }}+F_{\text {intra. }}+\widetilde{F}_{\text {inter } \|}$

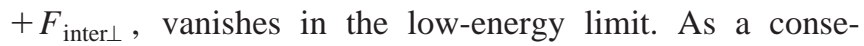
quence of this fact we have seen that, for an open system that is allowed to exchange particles with a charge reservoir, there is a certain range of fillings forbidden above and below the Van Hove singularity. This has the property of attracting the Fermi level for the corresponding values of the total charge in the mentioned range, as the system reaches then its lowest energy when the Fermi energy is at the singularity.

The mechanism of pinning to the Van Hove singularity could be relevant to explain some of the properties of the hole-doped copper-oxide superconductors. Angle-resolved photoemission experiments, ${ }^{24}$ as well as quantum Monte Carlo computations for the $t-J$ and Hubbard models, ${ }^{25}$ have shown very flat portions of the quasiparticle dispersion at the boundary of the Brillouin zone. In different compounds, the Fermi level has been estimated to be very close to saddle points of the band. These observations have been contested by the fact that the evidence for quasiparticles does not appear quite clear, given the broad peak of the spectral weight near the Fermi energy. However, the reduction in the quasiparticle weight is another of the consequences which derives from the interaction of electrons near a Van Hove singularity. It has been shown that the electron wave function is strongly renormalized in these circumstances. ${ }^{11}$ Although the quasiparticle description does not lose its validity, there is a strong attenuation of the quasiparticle pole as the Van Hove singularity is approached, and the normal state of the system adheres to the so-called marginal Fermi liquid behavior. ${ }^{26}$

The greater stability attained when the Fermi level approaches the Van Hove singularity could have experimental signatures in other systems that are essentially twodimensional and may exchange particles with the environment. Interfaces like $\mathrm{Sn} / \mathrm{Ge}(111)$ have been much studied recently, as they show a remarkable phase transition with the formation of a surface charge-density-wave in the lowtemperature phase. ${ }^{27}$ Photoemission experiments have shown the appearance of a very flat conduction band, ${ }^{28}$ which is found at an energy sensibly smaller than predicted by conventional band calculations. An important property is that the system remains metallic across the transition, what makes plausible the description by means of weak coupling RG methods. It has been proposed actually that the main features of the interface, including the loss of spectral weight below the transition and the formation of the charge-density-wave structure, can be explained by the effect of pinning at a Van Hove singularity that is present in the conduction band of the 2D system. ${ }^{29}$

We remark finally that the stability of the Van Hove filling may result in the effect of phase separation over a wide range of nominal filling levels above and below the Van Hove singularity. ${ }^{23}$ This effect has to be realized when the system is in contact with a sufficiently large reservoir, as we have shown in the paper. A most important question would be to ascertain, from the experimental point of view, to what extent the perovskite structure of the high- $T_{c}$ materials may lead to the pinning mechanism we have proposed, and whether the phase separation associated with it may bear some relation to that observed in the form of stripes in the underdoped cuprates. 
${ }^{1}$ J. Labbé and J. Bok, Europhys. Lett. 3, 1225 (1987); J. Friedel, J. Phys. (Paris) 48, 1787 (1987); 49, 1435 (1988); R. S. Markiewicz and B. G. Giessen, Physica C 160, 497 (1989); P. C. Pattnaik et al., Phys. Rev. B 45, 5714 (1992).

${ }^{2}$ A complete review of the Van Hove scenario for high- $T_{c}$ superconductivity is R. S. Markiewicz, J. Phys. Chem. Solids 58, 1179 (1997).

${ }^{3}$ H. J. Schulz, Europhys. Lett. 4, 609 (1987); P. Lederer, G. Montambaux, and D. Poilblanc, J. Phys. (Paris) 48, 1613 (1987); J. E. Dzyaloshinskii, Pis'ma Zh. Éksp. Teor. Fiz. 46, 97 (1987) [JETP Lett. 46, 118 (1987)].

${ }^{4}$ J. González, F. Guinea, and M. A. H. Vozmediano, Europhys. Lett. 34, 711 (1996); cond-mat/9502095.

${ }^{5}$ D. Zanchi and H. J. Schulz, Phys. Rev. B 54, 9509 (1996); D. Z. Liu and K. Levin, Physica C 275, 81 (1997).

${ }^{6}$ N. Furukawa, T. M. Rice, and M. Salmhofer, Phys. Rev. Lett. 81, 3195 (1998).

${ }^{7}$ J. V. Alvarez, J. González, F. Guinea, and M. A. H. Vozmediano, J. Phys. Soc. Jpn. 67, 1868 (1998).

${ }^{8}$ C. J. Halboth and W. Metzner, Phys. Rev. B 61, 7364 (2000).

${ }^{9}$ C. Honerkamp, M. Salmhofer, N. Furukawa, and T. M. Rice, cond-mat/9912358.

${ }^{10}$ Careful analyses have been also carried out in models with flat patches of the Fermi surface by N. Furukawa and T. M. Rice, J. Phys.: Condens. Matter 10, L381 (1998); J. Kishine and K. Yonemitsu, Phys. Rev. B 59, 14823 (1999); F. Vistulo de Abreu and B. Doucot, cond-mat/0007463.

${ }^{11}$ J. González, F. Guinea, and M. A. H. Vozmediano, Nucl. Phys. B 485, 694 (1997).

${ }^{12}$ J. González, F. Guinea, and M. A. H. Vozmediano, Phys. Rev. Lett. 84, 4930 (2000).
${ }^{13}$ R. S. Markiewicz, J. Phys.: Condens. Matter 2, 665 (1990).

${ }^{14}$ C. J. Halboth and W. Metzner, cond-mat/0003349.

${ }^{15}$ R. Shankar, Rev. Mod. Phys. 66, 129 (1994).

${ }^{16}$ J. Polchinski, in Proceedings of the 1992 TASI in Elementary Particle Physics, edited by J. Harvey and J. Polchinski (World Scientific, Singapore, 1992).

${ }^{17}$ H. Q. Lin and J. E. Hirsch, Phys. Rev. B 35, 3359 (1987).

${ }^{18}$ W. Metzner, C. Castellani, and C. di Castro, Adv. Phys. 47, 3 (1998).

${ }^{19}$ H. J. Schulz, in Correlated Electron Systems, edited by V. J. Emery (World Scientific, Singapore, 1993).

${ }^{20} \mathrm{We}$ note that the use of different conventions makes the coupling $U_{\text {back }}$ in Ref. 7 to correspond to the coupling $E_{\text {inter } \perp}$ defined in the present paper.

${ }^{21}$ This value is also obtained from Ref. 17 when the proper normalization of the particle-hole susceptibility at vanishing momentum is taken into account.

${ }^{22}$ S. Sorella, R. Hlubina, and F. Guinea, Phys. Rev. Lett. 78, 1343 (1997).

${ }^{23}$ Related ideas regarding phase separation have been discussed in Ref. 13.

${ }^{24}$ Z.-X. Shen et al., Science 267, 343 (1995).

${ }^{25}$ N. Bulut, D. J. Scalapino, and S. R. White, Phys. Rev. Lett. 72, 705 (1994); E. Dagotto, A. Nazarenko, and M. Boninsegni, ibid. 73, 728 (1994); E. Dagotto, A. Nazarenko, and A. Moreo, ibid. 74, 310 (1995).

${ }^{26}$ C. M. Varma et al., Phys. Rev. Lett. 63, 1996 (1989).

${ }^{27}$ J. M. Carpinelli et al., Phys. Rev. Lett. 79, 2859 (1997).

${ }^{28}$ A. Goldoni and S. Modesti, Phys. Rev. Lett. 79, 3266 (1997).

${ }^{29}$ J. González, Phys. Rev. B 62, 6928 (2000). 